\title{
Hoppe-Urteil: Ein Beitrag zur Beschleunigung
}

I0 Jahre gegen Werner Hoppe. Eine runde Zahl. Für das Schwurgericht war nach 33 Verhandlungstagen alles klar. Das Wort $* Z$ weifel «, das am häufigsten gebrauchte in den Plädoyers von Staatsanwalt und Verteidigung, kam im Urteil nicht vor. Es stand alles fest.

Feststand, daß Werner Hoppe des dreifachen versuchten Totschlags schuldig ist. 25 Minuten brauchte das Gericht zur Urteilsbegründung - oder $81 / 3$ Minuten pro versuchten Totschlag. Staatsanwalt und Verteidigung hatten sich offenbar nur mit nebensächlichen Punkten auseinandergesetzt, wenn sie insgesamt ro Stunden plädiert hatten. Das Gericht ging deshalb auf die von ihnen erörterten Probleme gar nicht erst ein: Was klar ist, kann man höchstens zerreden. Deshalb brauchten 9 Richter auch nur $3^{1 / 2}$ Stunden, um das Urteil zu beraten, bevor sie sich wie an jedem Tag in dem Streifenwagen der Polizei wieder nach Hause fahren ließen.

Feststand das Urteil offenbar schon, als ein Polizist Hoppes Ausruf bei der Festnahme zitierte: "Schade, daß ich von euch Schweinen nicht ein paar umgelegt habe.* Mit diesem Satz begannen die Urteilsgründe - unter diesem Satz stand das ganze Urteil - dieser Satz machte offenbar auch eine Beratung überflüssig. Und Ortwin Löwa befand im NDR: »Wer an den 33 Verhandlungstagen teilgenommen hatte, glaubte bei der Urteilsbegründung, er wäre in einem anderen Prozeß gewesen." Auf die Beweisaufnahme, die in der Urteilsbegründung nur kursorisch als Pflichtübung gestreift wurde, kann sich das Urteil denn auch in der Tat schwerlich stützen:

*Zumindest viermal « soll Hoppe geschossen haben. Zwar fehlten ihm nur höchstens 2 Patronen in der Waffe, und in der Hosentasche hatte er ein volles Magazin. Dies, so der Vorsitzende, »läßt nur den einen möglichen Schluß zu, daß er vorher aus einem dritten Magazin geschossen und dies dann weggeworfen hat «. - Kein Wort hiervon fiel in der Beweisaufnahme. Das metallene Magazin, groß wie ein Pistolengriff, fand man nicht einmal mit Minensuchgeräten... Der Waffensachverständige hatte überdies die Pulverrückstände untersucht und war auf »höchstens 3 Schüsse « gekommen.

Mündungsfeuer kann man bei Tageslicht nicht einmal auf $40 \mathrm{~cm}$ sehen. Das hat der Waffensachverständige geklärt.

Polizist I will es auf $37 \mathrm{~m}$ gesehen haben. Das Gericht: der Zeuge hat das Wort »Mündungsfeuer" nicht gewählt, um damit zu sagen, daß er das Mündungsfeuer gesehen habe - das sei ja auch unsichtbar -, sondern den Rauchkegel. Grund: Nur dieser sei sichtbar.

Polizist 2 - er hat im selben Zimmer wie Polizist I anschließend seinen Bericht vorbereitet - will beim selben Schuß ebenfalls auf $37 \mathrm{~m}$ hellgelbes Mündungsfeuer gesehen haben. Gericht: Das macht den Zeugen nicht unglaubwürdig. Also: Hoppe hat geschossen.

Polizist 3 will auf 4 bis $6 \mathrm{~m}$ Mündungsfeuer gesehen haben. Gericht: der Zeuge hat das Wort "Mündungsfeuer « nur gewählt, um zu erläutern, was er gesehen habe. Das erklärt vieles.

Polizist 4 hat den Schuß selber nicht gesehen. Auch kein Mündungsfeuer. Gericht: Das macht ihn glaubwürdig. Also: Hoppe hat geschossen.

Zusatz: Polizist 3 war mit Polizist 4 im Polizei-VW hinter den Sitzen in Dekkung gegangen und hat den Schuß Bruchteile von Sekunden später gehört. Das Gericht hat nicht etwa erklärt: der Zeuge hat das Wort "gesehen« nur gewählt, 
um zu erläutern, daß er das Mündungsfeuer gehört habe. Das macht ihn glaubwürdig.

Hoppe hat jedesmal mit Tötungsvorsatz geschossen. Beim ersten Schuß ergibt sich das daraus, daß er aus 4 bis $5 \mathrm{~m}$ auf die hinter den VW-Sitzen in Dedkung gegangenen Polizisten gezielt und das Dach getroffen hat. Die Gradabweichung von $35 \mathrm{Grad}$ erklärt das Gericht mit der möglichen Ungeübtheit des Schützen. Denn der Waffensachverständige hatte erklärt: »Der erste Schuß gehe immer dahin, wo man ihn haben will «.

Woher das Gericht diese Erkenntnisse - wenn schon nicht aus der Hauptverhandlung - gewonnen hat, zeigt ein Blick in die Anklageschrift: "Es ist dem Beschuldigten möglich gewesen, weitere Patronen vor- bzw. nachzuladen oder aber ein leergeschossenes Magazin auszuwechseln und wegzuwerfen. "Daß der Staatsanwalt diesen Standpunkt nach der Beweisaufnahme selber aufgegeben hat. kam für das Gericht offensichtlich zu spät. Das Urteil stand offenbar von Anfang an fest.

$\mathrm{Da}$ das Urteil eines bundesrepublikanischen Gerichts so deutlich erkennen ließ, wie wenig es auf die Hauptverhandlung ankommt, war neu. Verteidiger Reinhard ("Ich halte das nicht mehr aus") bat um eine Unterbrechung, um sich »vor Reaktionen zu schützen, die ggf. standeswidrig sein kïnnten«. Worauf das Urteil in Wahrheit beruht, verlautete in Kreisen der politischen Staatsanwaltschaft: "Ein politisches Urteil.« Offenbar sind in der Tat andere Maßstäbe für die "Wahrheitsfindung" angewandt worden als sonst. Noch bevor das Urteil gesprochen war, hatte Verteidiger Hannover die in politischen Prozessen praktizierte Definition von Straftatbeständen am Beispiel von Tötungsdelikten aufgedeckt: "Wer einen anderen Menschen im Interesse der herrschenden Klasse tötet, bleibt straflos. Wer gegen die Intcressen der herrschenden Klasse töter, ist hart zu bestrafen. "Dieser Satz erscheint als der Schlüssel für die Verurteilung auch von Werner Hoppe. Es selber hat im Prozeß keinen Zweifel daran gelassen, daß er die bestehende Gesellschaftsordnung mitsamt ihrer Justiz ablehnt. Uberdies haben ihn Anklage und Presse bewußt in die Nähe der RAF gerückt. Zwar hat er nicht getötet, es nach der Beweisaufnahme auch nicht versucht. Aber er mußte als Gegner des Systems bestraft werden. Auch für Werner Hoppe ist es ein politisches Urteil. Zu seinem Verteidiger Reinhard: „Ein Urteil, das sie über die Revolution fällen wollen.*

Systemfeindliche Gesinnung als Ersatz für den Beweis einer Straftat - auf dieser Grundlage müssen also nicht nur die nächsten Prozesse gegen RAF-Mitglieder eingeschätzt werden. Längst hat auch das Vorgehen gegen politische Gruppen unter dem Vorwand staatsanwaltschaftlicher Ermittlungsverfahren geheimpolizeilichen Charakter und damit eine unbegrenzte Fülle neuer Möglichkeiten erhalten. In Hamburg genügte jüngst ein bei Gudrun Ensslin gefundener Ausweis, um außer der Wohnung der in dem Ausweis genannten Person gleich noch das Büro des Kommunistischen Bundes (KB) mit zu durchsuchen und es einer fotografischen Registrierung zu unterziehen.

Eine neue Qualität hat das Hoppe-Urteil dennoch: das Ignorieren des Beweisergebnisses ist geradezu dreist, die Umstände: $3^{1 / 2}$ Stunden Beratung, 25 Minuten Begründung nach 33 Verhandlungstagen und 700 Seiten Tonbandprotokoll eklatant. Fanden andere Gerichte wenigstens noch einen Vorwand im Schlußwort einiger Angeklagter, ist hier ndie Entlarvung des Terrors hinter dem Rechtsstaatsgeschwätz " (Hoppe) offenbar geworden. Hoppe selber hat sich zu dem Urteil geäußert, »daß die Demaskierung der Justiz perfekt war, daß die demokratischen Gewänder objektiv weg sind und für sehr viele Menschen subjektiv 
Löcher bekommen haben «. Die Justiz habe aber auch keine andere Wahl gehabt: "Entweder stellt die Justiz die Illegalität der Bullen fest, oder sie begibt sich selbst in die Illegalität. «

Die Öffentlichkeit hat den Unterschied bemerkt. Nicht nur, daß das »Bombenurteil « (Frankfurter Rundschau) bei den Presseberichterstattern selber wie eine Bombe einschlug, nicht nur daß selbst Polizisten im Gerichtssaal zusammenzuckten, daß die Richter beim letzten Satz mit fliegendem Start und wehenden Uniformen ins Beratungszimmer flüchteten und die Staatsanwaltschaft das Urteil "schädlich" fand: Eine Flut des Protests erreichte die Verteidiger, an Werner Hoppe selber schickte u. a. die Belegschaft einer Kölner Firma Solidaritätsbekundungen. Die neue Qualität des Urteils nicht erkannt hat jedoch diejenige Presse, "die bei einer Kritik stehenbleibt, die nicht die Willkür und den Terror sieht, nicht die gesamte Faschisierung, sondern die nur den Fleck auf dem Rechtsstaat< kritisiert und sich nur ausläßt: >6 Jahre wären doch genug gewesen<.

Peggy Parrass

\section{Zentrales Informationsbüro (ZIB)}

Im März dieses Jahres haben sich unter dem Namen ২Zentrales Informationsbüror Rechtsanwälte, die insbesondere politische Strafverteidigungen übernehmen und Juristen zusanmengeschlossen, um ihre Arbeit durch gegenseitige Information zu erleichtern und zu koordinieren.

Zunächst wurde ein umfangreicher Katalog der Rechtsbereiche aufgestellt, die für diese Anwaltspraxen besonders relevant sind, nämlich u. a. Straf- und Strafprozeßrecht, Verfassungs- und Verwaltungsrecht, Schul- und Hochschulrecht, Kriegsdienstverweigerungs- und Ersatzdienstrecht, Ausländerrecht, Mietrecht, Arbeitsrecht, Sozialrecht, Versammlungsrecht.

Inzwischen hat die Sammlung des jeweiligen einschlägigen Materials (Entscheidungen, Aufsätze, etc.), das von allen Beteiligten und der Zentralstelle in Heidelberg gesammelt und von letzterer aufgearbeitet wird, begonnen.

Die Auswertung und Weitergabe dieses Materials erfolgt kontinuierlich an alle Mitglieder des ZIB. Zusätzlich kann unter Angabe des gesuchten Problembereiches weiteres Material vom ZIB oder mit dessen Hilfe von einzelnen Mitgliedern angefordert werden.

Barbara Dietrich

\section{Wirtschaftskriminalität - am Beispiel der Kundenkreditbank}

I.

Aus den Kreditbedingungen für Abzahlungsgeschäfte der Kundenkreditbank KGaA., Düsseldorf (Fassung vom I. 8. 197I):

7. Der Restsaldo ist zur sofortigen Rückzahlung fällig, wenn die Kreditnehmer mit einer Rate länger als 20 Tage in Verzug geraten.

17. Gerichtsstand für alle Streitigkeiten der Bank gegen Kreditnehmer, Mitverpflichtete, Bürgen oder Garantiegeber ist der Sitz der Niederlassung der KKB ... 